\title{
The Effects of Calcination on Mineral Composition and Physical Properties of Limestones and Oyster Shells Derived from Different Sources
}

\author{
Khalil $^{1^{*}}$, Ridho Kurniawan Rusli ${ }^{1}$, and Andri ${ }^{2}$ \\ ${ }^{I}$ Department of Animal Nutrition and Feed Technology, Faculty of Animal Science, Andalas University Campus II Payakumbuh, Payakumbuh 26224, \\ West Sumatra. Indonesia \\ ${ }^{2}$ Department of Livestock Business and Development, Faculty of Animal Science, Andalas University, Padang 25163, West Sumatra, Indonesia \\ *Corresponding author's Email: khalil@ansci.unand.ac.id; (DORCiD: 0000-0001-6359-8634
}

\begin{abstract}
Limestones and oyster shells are normally used in raw or roasted meal form in the livestock diet. Calcination is intended to improve the mineral concentration and physical characteristics of limestones and oyster shells which vary based on different chemical compositions, textures, and impurities of their types and habitats. The present research aimed to study the effects of calcination on mineral composition and the physical properties of limestones and oyster derived from various sources. Limestone samples from three local limestone mining and oyster shell samples from three shellfish species were calcined by burning at a temperature of $800-1000^{\circ} \mathrm{C}$. The calcined products were analyzed for mineral content $(\mathrm{Ca}, \mathrm{P}, \mathrm{Mg}, \mathrm{Cu}, \mathrm{Zn}$, and $\mathrm{Mn}$ ), physical properties (bulk density, tapped density, specific density, and angle of repose), and particle size. Results indicated that calcination had no significant effect on $\mathrm{Ca}$ and $\mathrm{P}$ concentrations but reduced micro mineral concentration. Limestones had a higher $\mathrm{Mg}$ concentration than that of oyster shells, and calcination increased $\mathrm{Mg}$ concentration. Calcined oyster shells had higher densities, percentages of fine particles, and lower angles of response. The results suggested that the type of limestones and oyster shells could determine their thermal decomposition properties.
\end{abstract}

Keywords: Calcination, Limestone, Mineral composition, Oyster shell, Physical property

\section{INTRODUCTION}

Limestone-based carbonate and waste oyster shells are widely used as sources of Calcium in livestock diet in the West Sumatra region of Indonesia due to their availability and inexpensiveness. The local limestone meals are produced from diverse natural carbonate rocks as part of the Bukit Barisan cluster. The mineral composition and macroscopical characteristics of limestones show considerable variations due to their diversity in Calcium (Ca) and $\mathrm{Magnesium}(\mathrm{Mg})$ contents, crystalline structure, and natural impurities (Kiliç, 2013). Limestone meal is usually labeled and sold as calcite $(\mathrm{CaCO} 3)$ and dolomite $\left(\mathrm{CaMg}\left[\mathrm{CO}_{3}\right]_{2}\right)$ for agricultural lime and animal feed considering that the ratio of $\mathrm{Ca}$ to $\mathrm{Mg}$ $(\mathrm{MgCO} 3 / \mathrm{CaCO} 3)$ varies depending on the type of limestone. Calcite is a light-colored white limestone, whereas dolomite is a light to dark brown limestones (Khalil and Anwar, 2007). The local calcite derived from Kamang, Agam district, West Sumatra, Indonesia, had also a high content of micro minerals (manganese, selenium, and iron, Khalil and Anwar, 2007). The use of the local limestones in the laying hen diet has a beneficial effect on egg production, eggshell quality, and bone mineralization (Khalil and Anwar, 2008).

The province of West Sumatra, Indonesia, located along the coast of the Hindian ocean, also abounds with bivalve shellfish living in various fresh water and terrestrial habitats of lakes, rivers, estuarine, and marine. Oyster shells derived from freshwater and marine, which represented up to $56 \%$ of the total body weight of the shellfish and contained Ca of about 31-36\%, exhibited different sizes and physical characteristics (Khalil et al., 2018). The shell could be processed in different forms of raw coarse, fine, or roasted for meals. However, the use of fine grinding and roasting of mussel shells in the diet of laying quails had no significant effect on egg production, eggshell quality, and feed utilization efficiency (Khalil et al., 2018). Therefore, it is of utmost importance to propose a suitable method for processing limestones and oyster shells in animal nutrition to enhance their food intake capacity and growth.

Calcination is a process through which mineral decomposition and the physical properties of limestones and oyster shells are upgraded using high temperatures $\left(\geq 700^{\circ} \mathrm{C}\right)$ in thermal treatment (Kiliç, 2013; Ha et al., 2019). The primary purpose of calcination is to eliminate water and organic matters and convert all or part of the calcium carbonate present into calcium dioxide ( $\mathrm{CaO}$, Özer, 2003). Calcination increased essential mineral concentration, finer particle percentage, and surface area (Özer, 2003; Ha et al., 2019), which can promote the food intake, digestibility, and absorption of the 
essential mineral. Therefore, the calcined products might have better nutritional value, especially for young poultry and livestock animals that need minerals in small amounts and still have low feed intake capacity and nutrient digestibility. Calcium is an essential macromineral, which should be necessarily supplied in animals' diet, especially during earlier phases of the growth period as a robust skeleton is required to support body mass. Some physical characteristics, such as bulk density, tapped density, specific density, and particle size are related to the nutritional values of mineral feed since they determine the intake capacity, digestibility, and absorption in the digestive tract. Calcined oyster shells had also potent antibacterial activity ( $\mathrm{Li}$ et al., 2014; Yao et al., 2014), which is mainly related to the alkaline effect caused by the hydration of $\mathrm{CaO}$ (Yao et al., 2014). The calcined limestones and oyster shells might be potentially used as a functional mineral for the improvement of feed storage stability and health status of young animals.

According to Boynton (1980), numerous factors determine the thermal decomposition quality of limestones and oyster shells, including crystallinity degree, types and quantities of impurities, as well as calcination duration and temperature. Limestones derived from various sources differ considerably in their chemical compositions, physical structure, and thermal decomposition properties (Kiliç, 2013). There is an optimum calcination temperature and rate of heating for each type of limestones that can only be determined by conducting an experiment (Kantiranis et al., 1999). The size and physical characteristics of oyster shells vary according to habitats and species. Larger body sizes of mussels and seashells tend to have stronger shell properties. Therefore, it is hypothesized that there is a difference in the mineralogical composition, physical properties, and particle size distribution of calcined products of limestones and oyster shells which can be ascribed to the different types, sizes, and thermal behaviors of limestones and oyster shells during calcination. The objective of the present study was to determine the calcination impact of different limestone and oyster shell types derived from different habitats on mineral composition, physical properties, and particle size and also compare the thermal decomposition characteristics of the two materials.

\section{MATERIALS AND METHODS}

Limestone samples were collected from three local rock mining companies that mined and processed different types of natural rock deposits by crushing and grinding them into calcite and dolomite flour. Two companies produce dolomite flour (Halaban and Palupuh) which contained Ca and Mg. Another company (Kamang) produces calcite flour containing high $\mathrm{Ca}$ and very low $\mathrm{Mg}$ (calcite flour).

Samples of three types of shells with different sizes and hardness were derived from fresh water and seashell. There were lake mussel (Corbicula moltkiana), blood clams (Anadara granosa), and pond mussels (Pilsbryoconcha exilis). Lake mussels were purchased in the intact form of about $100 \mathrm{~kg}$ from fishers of Singkarak Lake (Tanah Datar district), Indonesia. Pond mussels of about $100 \mathrm{~kg}$ were collected from fishponds located in Kamang Mudik sub-district of Agam district, West Sumatra, Indonesia. The shell part of mussels was separated and prepared according to the procedures described by Khalil et al. (2018). Samples of the clamshell of about $50 \mathrm{~kg}$ were derived from a seafood stall located at the coastal area of Tanjung Mutiara subdistrict, Agam district of West Sumatra.

The limestones and shell samples were thermally calcinated by burning them in a modified furnace made from metal drums. Calcination lasted for about 48-72 hours with a temperature of $800-900^{\circ} \mathrm{C}$ until the limestones and shells turned white and brittle. The calcined products were then ground into meal form. Three samples of each calcined limestone and oyster shell meals along with uncalcined or non-calcined limestone and oyster shell meals were taken and then analyzed for minerals, physical properties, and particle size. The analyzed mineral content included $\mathrm{Ca}, \mathrm{P}, \mathrm{Mg}, \mathrm{Cu}$, $\mathrm{Zn}$, and Mn using a spectrophotometer (AAS) according to the standard method described by AAS (1996).

The analyzed physical properties included bulk density, tapped density, specific density, and angle of response. Bulk and tapped densities were measured by a 100-mm graduated cylinder using the method described by Ruttloff (1981). Bulk density is the ratio of the weight of the sample (w) with the volume of space filled by the sample (v1), while tapped density is the ratio of the weight of the sample (w) with the volume of space filled by the sample after compacted (v2). Bulk and tapped densities $(\mathrm{g} / \mathrm{ml})$ were calculated using w/v1 and w/v2 in $\mathrm{g} / \mathrm{ml}$ formulas, respectively. Specific density was measured using a 25-ml Piknometer Pyrex. The angle of repose was measured using a cylinder placed on a flat surface of beaker glass according to a method described by Geldart et al. (2006).

Particle size was determined by sieve analysis using a sieve shaker Retsch VS 1000 (Retch GmbH, Germany). About $300 \mathrm{~g}$ samples were sieved for 10 minutes using a set of sieves with discrete size ranges of $500-1000 \mu \mathrm{m}$ classified into four different levels of coarse $(>1000 \mu \mathrm{m})$, medium $(1000-710 \mu \mathrm{m})$, fine $(710-500 \mu \mathrm{m})$, and very fine $(<500 \mu \mathrm{m})$. The particle number of detained parts in each sieve was weighed, and the percentage of each particle size 
was then calculated. The percentage of each particle size was the weight of detained part divided with the total weight of the sample and multiplied by $100 \%$.

The data obtained were expressed as mean and standard deviation (mean \pm SD) and statistically analyzed using two-way variance analysis (ANOVA) in a completely factorial randomized design using IBM SPSS Software version 7. The Least Significant Difference (LSD) was applied to separate means. Significant differences were considered at $\mathrm{p}<$ 0.05 (Steel et al., 1997).

\section{RESULTS}

\section{Effect of calcination on mineral composition}

Data on the effect of calcination on the macro and trace minerals of limestones and oyster shell meals derived from different sources are presented in Tables 1 and 2. The limestones of Kamang and Halaban had significantly higher Ca than that of Palupuh's limestones $(\mathrm{p}<0.05)$. On the other hand, Palupuh limestones contained considerably high Mg of 123.2-145.4 g/kg, compared to the other types of limestones (1.7-9.5 g/kg) and oyster shells (0.1-3.6 g/kg), which may be ascribed to its high $\mathrm{Mg}$ dolomite $(\mathrm{p}<0.05)$.

There was no significant difference in the Ca concentration of oyster shells derived from different habitats ( $\mathrm{p}>$ 0.5). The mean Ca content of the oyster shells $(421.6 \mathrm{~g} / \mathrm{kg})$ was slightly higher than that of limestones $(379.5 \mathrm{~g} / \mathrm{kg})$. The $\mathrm{Mg}$-concentration of $2.74 \mathrm{~g} / \mathrm{kg}$ of lake mussel was significantly higher than a pond and marine shells of $0.1 \mathrm{~g} / \mathrm{kg}$ (p < 0.05). There was no significant effect of calcination on Ca concentration by both limestones and shell ( $\mathrm{p}>0.05)$. Numerically, calcined limestones and lake mussels had higher $\mathrm{Ca}$ concentrations than the uncalcined products. The highest $\mathrm{Ca}$ level was observed in calcined Kamang limestones followed by calcined lake mussel shells. Phosphorus concentration in both limestones and oyster shells ranging from 0 to $0.12 \mathrm{~g} / \mathrm{kg}$ was very low and was not affected by the calcination process. However, calcination increased $\mathrm{Mg}$ concentration in both limestones and oyster shells, especially in Palupuh limestones $(\mathrm{p}<0.05)$.

Palupuh limestones had significantly higher $\mathrm{Cu}, \mathrm{Zn}$, and $\mathrm{Mn}$ than Kamang's and Halaban's limestones. Moreover, $\mathrm{Cu}, \mathrm{Zn}$, and Mn were significantly higher in the Mussel shell than clamshell ( $\mathrm{p}<0.05$ ). Except for $\mathrm{Zn}$ of dolomite and oyster shells, calcination reduced trace mineral concentration. Calcined Palupuh limestones had a significantly higher $\mathrm{Zn}$ concentration than the uncalcined ones ( $\mathrm{p}<0.05$ ). Calcination significantly increased $\mathrm{Zn}$ concentration in all shells and the highest value was for lake mussels $(\mathrm{p}<0.05)$. Data on trace minerals showed high variation, especially by $\mathrm{Zn}$ and Mn of oyster shells.

\section{Effect of calcination on physical properties and particle size}

Tables 3 and 4 compared the data on physical properties and particle size distribution of calcinated and uncalcined limestones and shells, respectively. Uncalcined Halaban limestones had the highest bulk and tapped densities but the lowest angle of repose among the uncalcined limestones. Palupuh limestones had a significantly higher specific density, compared to the Kamang and Halaban limestones ( $\mathrm{r}$ 0.05). Lake mussel shells had the highest bulk density, but the lowest specific density and angle of repose. Clamshells had the highest tapped and specific densities, while the highest angle of repose was for the pond mussel shell. Except for tapped and specific densities of Palupuh's limestone, calcination significantly increased densities but decreased the angle of repose of limestones $(\mathrm{p}<0.05)$.

On the contrary, calcined shells had lower bulk and tapped densities but higher specific density and angle of repose than the uncalcined shells. The calcined mussel shell had the highest tapped densities and lowest angle of repose. Calcined limestones and oyster shells indicated nearly the same values regarding tapped and specific densities

Both uncalcined and calcined limestones were dominated by a very fine particle $(<500 \mu \mathrm{m})$ composed of more than $90 \%$. Palupuh's limestones possessed the highest portion of the coarse particles and the lowest percentage of very fine particles. There was no significant effect of calcination on the particle size of limestones $(\mathrm{p}>0.05)$. The percentage of the very fine particles of calcines $(<500 \mu \mathrm{m})$ was even lower than that of the uncalcined limestones $(\mathrm{p}<0.05)$. On the other hand, the coarse particles $(>1000 \mu \mathrm{m})$ percentage of oyster shell meals were comparable to the fine particle in all uncalcined oyster shell meals. Pond mussel shells had the highest percentage of coarse particles, followed by lake mussel and blood clam. Calcination significantly reduced the particle size of the shells. The percentage of the coarse particle of shells decreased from $27-48.5 \%$ of uncalcined meal to $1.4-2.8 \%$ of calcined products, while the portion of the very fine particle increased from $29-45 \%$ to $78-86 \%$ 
Table 1. The effects of calcination on the mineral composition of limestones derived from three different types of rocks

\begin{tabular}{|c|c|c|c|c|c|c|}
\hline \multirow{2}{*}{ Mineral } & & \multirow{2}{*}{ Products } & \multicolumn{3}{|c|}{ Source of limestones } & \multirow{2}{*}{ Mean } \\
\hline & & & Kamang & Halaban & Palupuh & \\
\hline \multirow{9}{*}{$\begin{array}{l}\text { Macro mineral } \\
(\mathrm{g} / \mathrm{kg})\end{array}$} & \multirow{3}{*}{ Calcium } & Uncalcined & $398.98 \pm 30.56^{\mathbf{c}}$ & $439.03 \pm 17.99^{\mathrm{a}}$ & $279.84 \pm 15.14^{\mathrm{e}}$ & $372.61 \pm 82.80$ \\
\hline & & Calcined & $428.41 \pm 10.43^{\mathrm{ab}}$ & $420.73 \pm 12.87^{b}$ & $310.05 \pm 2.24^{\mathrm{d}}$ & $386.40 \pm 66.23$ \\
\hline & & Mean & $413.69 \pm 20.81^{\mathrm{A}}$ & $429.88 \pm 12.93^{\mathrm{A}}$ & $294.94 \pm 21.36^{\mathrm{B}}$ & $379.51 \pm 73.68$ \\
\hline & \multirow{3}{*}{ Phosphorus } & Uncalcined & $0.12 \pm 0.03$ & $0.11 \pm 0.02$ & $0.11 \pm 0.01$ & $0.11 \pm 0.01$ \\
\hline & & Calcined & $0.12 \pm 0.02$ & $0.12 \pm 0.02$ & $0.11 \pm 0.01$ & $0.11 \pm 0.01$ \\
\hline & & Mean & $0.12 \pm 0.00$ & $0.12 \pm 0.01$ & $0.11 \pm 0.01$ & $0.11 \pm 0.01$ \\
\hline & \multirow{3}{*}{ Magnesium } & Uncalcined & $3.79 \pm 1.38^{\mathrm{d}}$ & $6.01 \pm 1.64^{\mathrm{cd}}$ & $123.20 \pm 9.16^{\mathrm{b}}$ & $44.33 \pm 68.31^{\mathrm{F}}$ \\
\hline & & Calcined & $1.68 \pm 0.08^{\mathrm{d}}$ & $9.51 \pm 3.88^{\mathrm{c}}$ & $145.43 \pm 3.45^{\mathrm{a}}$ & $52.20 \pm 80.83^{\mathrm{E}}$ \\
\hline & & Mean & $2.74 \pm 1.49^{\mathrm{C}}$ & $7.76 \pm 2.47^{\mathrm{B}}$ & $134.31 \pm 15.72^{\mathrm{A}}$ & $48.27 \pm 74.56$ \\
\hline \multirow{9}{*}{$\begin{array}{l}\text { Micro mineral } \\
(\mathrm{ppm})\end{array}$} & \multirow{3}{*}{ Cupper } & Uncalcined & $2.23 \pm 0.50$ & $1.62 \pm 0.38$ & $3.98 \pm 0.73$ & $2.61 \pm 1.22^{\mathrm{E}}$ \\
\hline & & Calcined & $1.69 \pm 0.60$ & $1.21 \pm 0.75$ & $2.16 \pm 0.40$ & $1.69 \pm 0.47^{\mathrm{F}}$ \\
\hline & & Mean & $1.96 \pm 0.38^{\mathrm{C}}$ & $1.41 \pm 0.29^{\mathrm{B}}$ & $3.07 \pm 1.29^{\mathrm{A}}$ & $2.15 \pm 0.84$ \\
\hline & \multirow{3}{*}{ Zink } & Uncalcined & $13.90 \pm 1.57^{\mathrm{c}}$ & $11.62 \pm 0.75^{\mathrm{d}}$ & $18.87 \pm 0.94^{\mathrm{b}}$ & $14.80 \pm 3.71$ \\
\hline & & Calcined & $8.09 \pm 2.23^{\mathrm{e}}$ & $8.42 \pm 2.00^{\mathrm{e}}$ & $22.96 \pm 1.62^{\mathrm{a}}$ & $13.16 \pm 8.49$ \\
\hline & & Mean & $11.00 \pm 4.10^{\mathrm{B}}$ & $10.02 \pm 2.26^{\mathrm{B}}$ & $20.92 \pm 2.89^{\mathrm{A}}$ & $13.98 \pm 6.03$ \\
\hline & \multirow{3}{*}{ Manganese } & Uncalcined & $37.16 \pm 5.39^{\mathrm{d}}$ & $57.06 \pm 10.95^{\mathrm{c}}$ & $245.12 \pm 3.98^{\mathrm{a}}$ & $113.11 \pm 114.76^{\mathrm{E}}$ \\
\hline & & Calcined & $19.56 \pm 3.81^{\mathrm{f}}$ & $26.49 \pm 4.02^{\mathrm{e}}$ & $129.43 \pm 2.75^{\mathrm{b}}$ & $58.49 \pm 61.53^{\mathrm{F}}$ \\
\hline & & Mean & $28.36 \pm 12.44^{\mathrm{B}}$ & $41.78 \pm 21.61^{\mathrm{B}}$ & $187.28 \pm 81.8^{\mathrm{A}}$ & $85.80 \pm 88.13$ \\
\hline
\end{tabular}

$\overline{a, b, c, d, e}$ values within a row with different superscripts differ significantly $(\mathrm{p}<0.05)$. ${ }^{\mathrm{A}, \mathrm{B}, \mathrm{C}}$ values within a row with different superscripts differ significantly $(\mathrm{p}<0.05)$. ${ }^{\mathrm{E}, \mathrm{F}}$ mean values of each micro mineral or macro mineral in the same column with different superscripts differ significantly $(\mathrm{p}<$ $0.05)$.

Table 2. The effects of calcination on the mineral composition of oyster shells derived from three different habitats

\begin{tabular}{|c|c|c|c|c|c|c|}
\hline \multirow{2}{*}{ Mineral } & & \multirow{2}{*}{ Products } & \multicolumn{3}{|c|}{ Oyster shells } & \multirow{2}{*}{ Mean } \\
\hline & & & Lake mussel & Blood clam & Pond mussel & \\
\hline \multirow{9}{*}{$\begin{array}{l}\text { Macro mineral } \\
(\mathrm{g} / \mathrm{kg})\end{array}$} & \multirow{3}{*}{ Calcium } & Uncalcined & $402.56 \pm 30.56$ & $457.65 \pm 55.61$ & $422.55 \pm 32.94$ & $427.59 \pm 27.88$ \\
\hline & & Calcined & $420.73 \pm 16.16$ & $420.60 \pm 19.41$ & $405.23 \pm 4.88$ & $415.52 \pm 8.91$ \\
\hline & & Mean & $411.65 \pm 12.85$ & $439.12 \pm 26.20$ & $413.89 \pm 12.25$ & $421.55 \pm 15.26$ \\
\hline & \multirow{3}{*}{ Phosphorus } & Uncalcined & $0.10 \pm 0.00$ & $0.10 \pm 0.01$ & $0.10 \pm 0.00$ & $0.10 \pm 0.00$ \\
\hline & & Calcined & $0.11 \pm 0.01$ & $0.10 \pm 0.01$ & $0.07 \pm 0.05$ & $0.10 \pm 0.02$ \\
\hline & & Mean & $0.11 \pm 0.01$ & $0.10 \pm 0.00$ & $0.09 \pm 0.02$ & $0.10 \pm 0.01$ \\
\hline & \multirow{3}{*}{ Magnesium } & Uncalcined & $3.79 \pm 1.38$ & $0.01 \pm 0.01$ & $0.08 \pm 0.00$ & $0.83 \pm 1.29$ \\
\hline & & Calcined & $1.68 \pm 0.08$ & $0.11 \pm 0.01$ & $0.11 \pm 0.02$ & $1.26 \pm 1.98$ \\
\hline & & Mean & $2.74 \pm 0.87^{\mathrm{A}}$ & $0.10 \pm 0.01^{\mathrm{B}}$ & $0.10 \pm 0.02^{\mathrm{B}}$ & $1.04 \pm 1.64$ \\
\hline \multirow{9}{*}{$\begin{array}{l}\text { Micro mineral } \\
(\mathrm{ppm})\end{array}$} & \multirow{3}{*}{ Cupper } & Uncalcined & $3.54 \pm 2.59$ & $2.79 \pm 0.81$ & $5.52 \pm 0.68$ & $3.95 \pm 1.41$ \\
\hline & & Calcined & $2.96 \pm 2.33$ & $2.79 \pm 0.46$ & $4.28 \pm 0.52$ & $3.34 \pm 0.82$ \\
\hline & & Mean & $3.25 \pm 0.41^{\mathrm{AB}}$ & $2.79 \pm 0.00^{\mathrm{B}}$ & $4.90 \pm 0.88^{\mathrm{A}}$ & $3.65 \pm 0.43$ \\
\hline & \multirow{3}{*}{ Zink } & Uncalcined & $13.09 \pm 5.69^{\mathrm{d}}$ & $9.81 \pm 1.40^{\mathrm{d}}$ & $15.54 \pm 2.72^{\mathrm{d}}$ & $12.81 \pm 2.88^{\mathrm{F}}$ \\
\hline & & Calcined & $191.34 \pm 15.07^{\mathrm{a}}$ & $38.43 \pm 3.44^{\mathrm{c}}$ & $99.35 \pm 12.00^{\mathrm{b}}$ & $109.71 \pm 76.98^{\mathrm{E}}$ \\
\hline & & Mean & $102.22 \pm 126.04^{\mathrm{A}}$ & $24.12 \pm 20.24^{\mathrm{C}}$ & $57.45 \pm 59.26^{\mathrm{B}}$ & $13.98 \pm 6.03$ \\
\hline & \multirow{3}{*}{ Manganese } & Uncalcined & $246.08 \pm 355.46$ & $39.94 \pm 5.12$ & $660.52 \pm 12.74$ & $315.51 \pm 316.06$ \\
\hline & & Calcined & $30.49 \pm 5.55$ & $57.61 \pm 1.40$ & $503.23 \pm 6.44$ & $197.11 \pm 265.46$ \\
\hline & & Mean & $138.29 \pm 152.45^{\mathrm{B}}$ & $48.77 \pm 12.49^{\mathrm{C}}$ & $581.88 \pm 111.22^{\mathrm{A}}$ & $256.31 \pm 285.48$ \\
\hline
\end{tabular}


Table 3. The effects of calcination on physical properties and particle size of limestones derived from three different sources

\begin{tabular}{|c|c|c|c|c|c|c|}
\hline \multirow{2}{*}{ Products } & & & \multicolumn{3}{|c|}{ Source of limestone } & \multirow{2}{*}{ Mean } \\
\hline & & & Kamang & Halaban & Palupuh & \\
\hline \multirow{12}{*}{$\begin{array}{l}\text { Physical } \\
\text { properties }\end{array}$} & \multirow{3}{*}{ Bulk density (g/ml) } & Uncalcined & $0.95 \pm 0.03^{\mathrm{f}}$ & $1.20 \pm 0.04^{\mathrm{d}}$ & $1.05 \pm 0.04^{\mathrm{e}}$ & $1.07 \pm 0.13^{\mathrm{F}}$ \\
\hline & & Calcined & $1.43 \pm 0.01^{\mathrm{b}}$ & $1.50 \pm 0.02^{\mathrm{a}}$ & $1.26 \pm 0.01^{\mathrm{c}}$ & $1.40 \pm 0.12^{\mathrm{E}}$ \\
\hline & & Mean & $1.19 \pm 0.34^{\mathrm{B}}$ & $1.35 \pm 0.21^{\mathrm{A}}$ & $1.16 \pm 0.15^{\mathrm{C}}$ & $1.23 \pm 0.10$ \\
\hline & \multirow{3}{*}{ Tapped density (g/ml) } & Uncalcined & $1.48 \pm 0.04^{\mathrm{e}}$ & $1.65 \pm 0.06^{b}$ & $1.59 \pm 0.03^{\mathrm{c}}$ & $1.57 \pm 0.09^{\mathrm{F}}$ \\
\hline & & Calcined & $1.79 \pm 0.00^{\mathrm{a}}$ & $1.76 \pm 0.00^{\mathrm{a}}$ & $1.54 \pm 0.00^{\mathrm{d}}$ & $1.70 \pm 0.14^{\mathrm{E}}$ \\
\hline & & Mean & $1.64 \pm 0.22^{\mathrm{B}}$ & $1.71 \pm 0.08^{\mathrm{A}}$ & $1.56 \pm 0.03^{C}$ & $1.64 \pm 0.07$ \\
\hline & \multirow{3}{*}{ Specific density $(\mathrm{g} / \mathrm{ml})$} & Uncalcined & $2.55 \pm 0.02^{\mathrm{e}}$ & $2.56 \pm 0.01^{\mathrm{de}}$ & $2.71 \pm 0.04^{\mathrm{a}}$ & $2.61 \pm 0.09^{\mathrm{F}}$ \\
\hline & & Calcined & $2.61 \pm 0.02^{\mathrm{c}}$ & $2.69 \pm 0.04^{\mathrm{a}}$ & $2.65 \pm 0.02^{\mathrm{b}}$ & $2.65 \pm 0.04^{\mathrm{E}}$ \\
\hline & & Mean & $2.58 \pm 0.04^{\mathrm{B}}$ & $2.62 \pm 0.10^{\mathrm{AB}}$ & $2.68 \pm 0.065^{\mathrm{A}}$ & $2.63 \pm 0.05$ \\
\hline & \multirow{3}{*}{ Angle of repose $\left(^{\circ}\right)$} & Uncalcined & $58.47 \pm 5.66^{\mathrm{a}}$ & $53.92 \pm 3.22^{b}$ & $58.78 \pm 1.91^{\mathrm{a}}$ & $57.06 \pm 2.72^{\mathrm{E}}$ \\
\hline & & Calcined & $49.10 \pm 0.50^{\mathrm{c}}$ & $42.43 \pm 2.83^{\mathrm{d}}$ & $48.51 \pm 0.52^{\mathrm{c}}$ & $46.68 \pm 3.70^{\mathrm{F}}$ \\
\hline & & Mean & $53.79 \pm 6.62^{\mathrm{A}}$ & $48.17 \pm 8.13^{\text {B }}$ & $53.65 \pm 7.26^{\mathrm{A}}$ & $51.87 \pm 3.20$ \\
\hline \multirow{12}{*}{$\begin{array}{l}\text { Particle } \\
\text { size }(\%)\end{array}$} & \multirow{3}{*}{ Coarse $(>1000 \mu \mathrm{m})$} & Uncalcined & $0.09 \pm 0.02^{\mathrm{e}}$ & $0.43 \pm 0.01^{\mathrm{c}}$ & $0.00 \pm 0.00^{\mathrm{f}}$ & $0.17 \pm 0.22^{\mathrm{F}}$ \\
\hline & & Calcined & $0.62 \pm 0.02^{b}$ & $0.16 \pm 0.03^{\mathrm{d}}$ & $2.16 \pm 0.05^{\mathrm{a}}$ & $0.98 \pm 1.04^{\mathrm{E}}$ \\
\hline & & Mean & $0.36 \pm 0.38^{\mathrm{B}}$ & $0.29 \pm 0.19^{\mathrm{C}}$ & $1.08 \pm 1.52^{\mathrm{A}}$ & $0.58 \pm 0.44$ \\
\hline & \multirow{3}{*}{ Medium $(1000-710 \mu \mathrm{m})$} & Uncalcined & $0.09 \pm 0.03^{\mathrm{e}}$ & $0.10 \pm 0.08^{\mathrm{d}}$ & $0.00 \pm 0.00^{\mathrm{f}}$ & $0.06 \pm 0.06^{\mathrm{F}}$ \\
\hline & & Calcined & $1.51 \pm 0.01^{\mathrm{b}}$ & $0.45 \pm 0.06^{\mathrm{c}}$ & $3.43 \pm 0.05^{\mathrm{a}}$ & $1.79 \pm 1.51$ \\
\hline & & Mean & $0.80 \pm 1.00^{\mathrm{B}}$ & $0.28 \pm 0.24^{\mathrm{C}}$ & $1.71 \pm 2.42^{\mathrm{A}}$ & $0.93 \pm 0.73$ \\
\hline & \multirow{3}{*}{ Fine $(710-500 \mu \mathrm{m})$} & Uncalcined & $0.14 \pm 0.08^{\mathrm{e}}$ & $0.18 \pm 0.01^{\mathrm{de}}$ & $0.00 \pm 0.00^{\mathrm{f}}$ & $0.11 \pm 0.10^{\mathrm{F}}$ \\
\hline & & Calcined & $4.21 \pm 0.14^{\mathrm{c}}$ & $4.26 \pm 0.54^{\mathrm{b}}$ & $5.40 \pm 0.11^{\mathrm{a}}$ & $4.62 \pm 0.67^{\mathrm{E}}$ \\
\hline & & Mean & $2.17 \pm 2.88^{\mathrm{B}}$ & $2.22 \pm 2.88^{\mathrm{AB}}$ & $2.70 \pm 3.82^{\mathrm{A}}$ & $2.36 \pm 0.29$ \\
\hline & \multirow{3}{*}{ Very fine $(<500 \mu \mathrm{m})$} & Uncalcined & $99.68 \pm 0.04^{\mathrm{b}}$ & $99.29 \pm 0.08^{c}$ & $100.00 \pm 0.00^{\mathrm{a}}$ & $99.66 \pm 0.36^{\mathrm{E}}$ \\
\hline & & Calcined & $93.65 \pm 0.14^{\mathrm{e}}$ & $95.13 \pm 0.61^{\mathrm{d}}$ & $89.02 \pm 0.12^{\mathrm{f}}$ & $92.60 \pm 3.19^{\mathrm{F}}$ \\
\hline & & Mean & $96.67 \pm 4.26^{\mathrm{B}}$ & $97.21 \pm 2.94^{\mathrm{A}}$ & $94.51 \pm 7.76^{\mathrm{C}}$ & $96.13 \pm 1.43$ \\
\hline
\end{tabular}

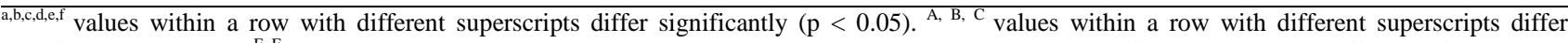
significantly $(\mathrm{p}<0.05)$. E, F mean values of each micro mineral or macro mineral in the same column with different superscripts differ significantly ( $\mathrm{p}<$ $0.05)$.

Table 4. The effects of calcination on physical properties and particle size of oyster shell meals derived from three different species

\begin{tabular}{|c|c|c|c|c|c|c|}
\hline \multirow{2}{*}{ Products } & & & \multicolumn{3}{|c|}{ Oyster shell } & \multirow{2}{*}{ Mean } \\
\hline & & & Lake mussel & Blood clam & Pond mussel & \\
\hline \multirow{12}{*}{$\begin{array}{l}\text { Physical } \\
\text { properties }\end{array}$} & \multirow{3}{*}{ Bulk density $(\mathrm{g} / \mathrm{ml})$} & Uncalcined & $1.43 \pm 0.02^{\mathrm{a}}$ & $1.40 \pm 0.01^{\mathrm{b}}$ & $1.36 \pm 0.01^{\mathrm{c}}$ & $1.40 \pm 0.04^{\mathrm{E}}$ \\
\hline & & Calcined & $1.33 \pm 0.01^{\mathrm{d}}$ & $1.10 \pm 0.01^{\mathrm{e}}$ & $0.96 \pm 0.01^{\mathrm{f}}$ & $1.13 \pm 0.19^{\mathrm{F}}$ \\
\hline & & Mean & $1.38 \pm 0.07^{\mathrm{A}}$ & $1.25 \pm 0.21^{\mathrm{B}}$ & $1.16 \pm 0.28^{\mathrm{C}}$ & $1.26 \pm 0.11$ \\
\hline & \multirow{3}{*}{ Tapped density (g/ml) } & Uncalcined & $1.63 \pm 0.02^{\mathrm{c}}$ & $1.68 \pm 0.01^{\mathrm{b}}$ & $1.60 \pm 0.03^{\mathrm{d}}$ & $1.63 \pm 0.04^{\mathrm{E}}$ \\
\hline & & Calcined & $1.76 \pm 0.03^{\mathrm{a}}$ & $1.59 \pm 0.01^{\mathrm{d}}$ & $1.47 \pm 0.01^{\mathrm{e}}$ & $1.61 \pm 0.15^{\mathrm{F}}$ \\
\hline & & Mean & $1.69 \pm 0.10^{\mathrm{A}}$ & $1.63 \pm 0.07^{\mathrm{B}}$ & $1.53 \pm 0.09^{\mathrm{C}}$ & $1.62 \pm 0.08$ \\
\hline & \multirow{3}{*}{ Specific density $(\mathrm{g} / \mathrm{ml})$} & Uncalcined & $1.74 \pm 0.05^{\mathrm{e}}$ & $2.73 \pm 0.05^{\mathrm{a}}$ & $2.30 \pm 0.02^{\mathrm{d}}$ & $2.26 \pm 0.50^{\mathrm{F}}$ \\
\hline & & Calcined & $2.57 \pm 0.05^{\mathrm{bc}}$ & $2.55 \pm 0.03^{\mathrm{c}}$ & $2.69 \pm 0.03^{\mathrm{a}}$ & $2.61 \pm 0.08^{\mathrm{E}}$ \\
\hline & & Mean & $2.15 \pm 0.59^{\mathrm{C}}$ & $2.64 \pm 0.12^{\mathrm{A}}$ & $2.50 \pm 0.28^{\mathrm{B}}$ & $2.43 \pm 0.25$ \\
\hline & \multirow{3}{*}{ Angle of repose $\left(^{\circ}\right)$} & Uncalcined & $40.97 \pm 1.36^{\mathrm{f}}$ & $43.57 \pm 1.65^{\mathrm{e}}$ & $45.67 \pm 0.58^{\mathrm{d}}$ & $43.41 \pm 2.35^{\mathrm{F}}$ \\
\hline & & Calcined & $51.81 \pm 1.98^{\mathrm{c}}$ & $56.45 \pm 1.94^{\mathrm{a}}$ & $54.30 \pm 1.23^{\mathrm{b}}$ & $54.19 \pm 2.32^{\mathrm{E}}$ \\
\hline & & Mean & $46.39 \pm 7.66^{\mathrm{B}}$ & $50.01 \pm 9.11^{\mathrm{A}}$ & $49.98 \pm 6.10^{\mathrm{A}}$ & $48.80 \pm 2.08$ \\
\hline \multirow{12}{*}{$\begin{array}{l}\text { Particle } \\
\text { size (\%) }\end{array}$} & \multirow{3}{*}{ Coarse $(>1000 \mu \mathrm{m})$} & Uncalcined & $31.65 \pm 0.90^{\mathrm{b}}$ & $27.75 \pm 0.34^{\mathrm{c}}$ & $48.51 \pm 0.64^{\mathrm{a}}$ & $35.97 \pm 11.03^{\mathrm{E}}$ \\
\hline & & Calcined & $2.82 \pm 0.26^{\mathrm{de}}$ & $2.61 \pm 0.13^{\mathrm{e}}$ & $1.42 \pm 0.08^{\mathrm{f}}$ & $2.29 \pm 0.76^{\mathrm{F}}$ \\
\hline & & Mean & $17.24 \pm 20.38^{\mathrm{B}}$ & $15.18 \pm 17.78^{\mathrm{C}}$ & $24.97 \pm 33.30^{\mathrm{A}}$ & $19.13 \pm 5.16$ \\
\hline & \multirow{3}{*}{ Medium $(1000-710 \mu \mathrm{m})$} & Uncalcined & $14.76 \pm 0.22^{\mathrm{a}}$ & $12.84 \pm 0.36^{\mathrm{b}}$ & $11.43 \pm 0.62^{\mathrm{c}}$ & $13.01 \pm 1.67^{\mathrm{E}}$ \\
\hline & & Calcined & $6.98 \pm 0.50^{\mathrm{d}}$ & $4.96 \pm 0.19^{\mathrm{e}}$ & $3.91 \pm 0.11^{\mathrm{f}}$ & $5.28 \pm 1.56^{\mathrm{F}}$ \\
\hline & & Mean & $10.87 \pm 5.50^{\mathrm{A}}$ & $8.90 \pm 5.58^{\text {B }}$ & $7.67 \pm 5.32^{\mathrm{C}}$ & $9.15 \pm 1.62$ \\
\hline & \multirow{3}{*}{ Fine $(710-500 \mu \mathrm{m})$} & Uncalcined & $16.52 \pm 0.13^{\mathrm{a}}$ & $14.72 \pm 0.20^{\mathrm{b}}$ & $10.62 \pm 0.21^{\mathrm{d}}$ & $13.96 \pm 3.02$ \\
\hline & & Calcined & $11.99 \pm 0.28^{\mathrm{c}}$ & $8.55 \pm 0.45^{\mathrm{e}}$ & $8.37 \pm 0.04^{\mathrm{e}}$ & $9.64 \pm 2.04$ \\
\hline & & Mean & $14.26 \pm 3.20^{\mathrm{A}}$ & $11.64 \pm 4.36^{\mathrm{B}}$ & $9.50 \pm 1.59^{\mathrm{C}}$ & $11.80 \pm 2.38$ \\
\hline & \multirow{3}{*}{ Very fine $(<500 \mu \mathrm{m})$} & Uncalcined & $37.07 \pm 0.63^{\mathrm{e}}$ & $44.68 \pm 0.52^{\mathrm{d}}$ & $29.44 \pm 0.33^{\mathrm{f}}$ & $37.06 \pm 7.62$ \\
\hline & & Calcined & $78.20 \pm 0.98^{c}$ & $83.88 \pm 0.76^{\mathrm{b}}$ & $86.29 \pm 0.22^{\mathrm{a}}$ & $82.79 \pm 4.15$ \\
\hline & & Mean & $57.63 \pm 29.09^{\mathrm{B}}$ & $64.28 \pm 27.71^{\mathrm{A}}$ & $57.87 \pm 40.21^{\mathrm{B}}$ & $59.93 \pm 3.77$ \\
\hline
\end{tabular}

$\overline{\mathrm{a}, \mathrm{b}, \mathrm{c}, \mathrm{d}, \mathrm{e}, \mathrm{f}}$ values within a row with different superscripts differ significantly $(\mathrm{p}<0.05) .{ }^{\mathrm{A}, \mathrm{B}, \mathrm{C}}$ values within a row with different superscripts differ significantly $(\mathrm{p}<0.05)$. E, F mean values of each micro mineral or macro mineral in the same column with different superscripts differ significantly ( $\mathrm{p}<$ $0.05)$.

\section{DISCUSSION}


The bioavailability and minor toxicity of $\mathrm{CaCO} 3$, along with its abundant resources and low production cost, have encouraged the widespread use of limestones and oyster shell wastes as a mineral supplement in the livestock diet (Khalil and Anwar, 2007; Jović et al., 2019). The results of the present study showed that Ca and Mg were the most important macrominerals contained in the local limestones and oyster shells. The $\mathrm{Ca}$ and $\mathrm{Mg}$ levels of uncalcined limestones in the current study were $379.5 \mathrm{~g} / \mathrm{kg}$ and $48.3 \mathrm{~g} / \mathrm{kg}$, respectively, which were much higher than the Ca (138.4 $\mathrm{g} / \mathrm{kg})$ and $\mathrm{Mg}(37.6 \mathrm{~g} / \mathrm{kg})$ of basalt rock used as soil fertilizer in the East Lampung reported by Hendronursito et al. (2019) or three Brazilian limestones reported by Silveira et al. (2020). The type of limestones strongly determined the concentration of $\mathrm{Mg}$, micro minerals, and physical properties (Khalil et al., 2011). Kamang and Halaban limestones were a high Ca type with a mean Ca content of more than $40 \%$, and when properly calcined, high-grade calcium oxide would be produced (Okonkwo and Adefila, 2013).

In the present study, the Ca content of uncalcined oyster shells was relatively constant at 40.2-45.8\%, which was slightly higher than the mean Ca level of 31.4-35.8\% of oyster shells derived from different habitats in West Sumatra reported by Khalil et al. (2018). The difference between limestones and oyster shells is in the levels of Ca, Mg, Mn, bulk density, and particle size. Uncalcined limestones possessed higher $\mathrm{Mg}$ and percentage of fine particle and lower bulk density than the oyster shells, while the oyster shells had higher in $\mathrm{Ca}, \mathrm{Mn}$, bulk density, and coarse particle than limestone. The different species and habitats of shellfish affect the $\mathrm{Mg}$ and trace mineral composition and particle size distribution.

The process of calcination of limestones and oyster shells was conducted using thermal decomposition properties (Kiliç, 2013). The thermal decomposition is intended to reduce the impurities and hence improve mineral concentration and bioavailability. Calcination is also designed to produce mineral products with finer and homogenous particle size and larger surface area, resulting in better mineral bioavailability and uniform physical properties. The calcined products, therefore, had distinctive nutritive values and better properties than the uncalcined or roasted meals. The present results indicated that calcination led to different effects on mineral composition, physical properties, and particle size distribution of limestones and oyster shells.

Generally, calcination increased macro minerals and density. On the other hand, particle size and trace mineral concentration decreased by calcination. Thermal decomposition is a temperature, chemical, and physical propertiesdependent process (Kiliç, 2013). This process started on the exterior surfaces and progressed inwards as the surrounding temperature increases, resulting in an increasing mass of $\mathrm{CaO}$, large surface area, and hence, high chemical reactivity (Kantiranis et al., 1999). The larger and harder the limestones and shell fragments, the higher the temperature required for decomposition.

The present data on trace minerals of $\mathrm{Zn}$ and $\mathrm{Mn}$ showed high variation, especially in the calcined oyster shells. The differences in chemical composition were due to the water filterer role of oysters and mussels. The mineral composition of the oyster shells was affected the water condition where these mussels grew (Kurunczi et al., 2001). There is a difference in calcination conditions between limestones and oyster shells for optimal decomposition. The calcination process of oyster shells was completed in a shorter time and at a lower temperature than limestones (Ha et al., 2019). The optimum calcination temperature for limestones and oyster shells was about $900^{\circ} \mathrm{C}$ and $750^{\circ} \mathrm{C}$, respectively, which was the temperature performed in traditional limekilns (Moropoulou et al., 2001; Park et al., 2021). According to Ha et al. (2019), the texture of calcite in limestones and oyster shells is more important than their chemical compositions that control the calcination temperature and specific surface area of the calcination products (Ha et al., 2019). They found that about $50 \%$ of the calcite in limestones was transformed to $\mathrm{CaO}$, but for oyster shells, more than $80 \%$ of calcite was transformed after 5 hours by calcination at $700^{\circ} \mathrm{C}$ (Ha et al., 2019). Another study on limestones showed that the samples did not decompose completely at $1050^{\circ} \mathrm{C}(8 \%$ of the calcite remained unaffected in the core) (Kantiranis et al., 1999). Another study on dolomite in Aceh confirmed that the $\mathrm{CaCO} 3$ decomposed into $\mathrm{CaO}$ by calcination at a temperature of $900{ }^{\circ} \mathrm{C}$ and produced homogenous particles size (Sari et al., 2013). However, some shell materials remained untransformed even at higher calcination temperatures and after a more extended treatment due to the sintered surface, which covers the rest of the parts (Lee et al., 2018).

Results of the present study showed that there was a significant increase in the Mg level of dolomite limestones probably due to the substantial weight loss. According to Kantiranis et al. (1999), the greater the MgCO3 component is in dolomitic limestone, the greater the weight loss would be. Magnesium in limestones determines the carbonate mineralogy of limestones and their physical characteristics, such as color, brightness, hardness, and decomposition properties (Kiliç, 2013). Since the $\mathrm{MgCO} 3 / \mathrm{CaCO} 3$ ratio varies based on the type of limestone, the decomposition temperature differs among various types of limestones (Kiliç, 2013). Carbonate limestones as a natural deposit rock contain various impurities, including SiO2, Al2 O3, and MgCO3 (Okonkwo and Adefila, 2013). Gupta et al. (2017) studied the effect of impurities on the quality of calcines reported that the presence of $\mathrm{Al}_{2} \mathrm{O}_{3}$ and $\mathrm{SiO}_{2}$ increases the activation energy, while the presence of $\mathrm{MgCO} 3$ lowers. 
The present results showed that the uncalcined oyster shells, especially pond mussels, contain a higher concentration of coarse particles. The presence of larger particles in the oyster shells led to a more heterogeneous distribution, compared to limestones. Physical characteristics are closely related to mineral composition, crystal structure, and particle size. Compared to limestones, the uncalcined oyster shells comprised a higher portion of coarse particles. The observed differences in particle size and distribution of particle size were related to the milling process. The grinding process in limestone mining companies consisted of several stages with different grinding systems, which produced more than $90 \%$ of very fine particles. The produced calcined and uncalcined oyster shells were ground using a laboratory hammer mill, resulting in only about $60 \%$ of the very fine particle. The larger particle size portion of oyster shells, compared to limestones, could also be associated with a higher hardness due to higher silica concentration (Hamester et al., 2012). The calcination of oyster shells significantly increased the portion of finer particles. It might contribute to better digestibility and absorption in the digestive tract, resulting in better nutritive values of the calcined product due to a larger surface area of finer particles (Ha et al., 2019). However, the increase in finer particle portions led to a significant increase in the angle of repose, and consequently a poorer flowability rate and less efficiency for bulk handling (Geldart et al., 2006).

The bulk and specific density of the limestones obtained in the current study were comparable to the values of three Brazilian limestones reported by Silveira et al. (2020). The present results indicated that the thermal treatment significantly increased the density values of both limestones and oyster shells. The specific density is closely related to intake capacity and mixture stability, therefore, higher density could result in higher mineral intake capacity. Ruttloff (1981) urges that the mixture of diet will be stable and not easily separated during handling and storage if the specific density value is not much different. The specific density, which is related to the texture characteristic of calcines, is smaller for oyster shells than for limestone; large mussels and seashells had structural solid properties. Oyster shells are composed of tiny prisms, foliated laths, and irregularly shaped folia of calcite with a loose texture, whereas the texture of calcite in limestones is crystalline and compact (Ha et al., 2019). The crystal structures of oyster shells primarily comprise aragonite and calcite, which have higher strengths and densities than limestones (Mosher et al., 2012). As Lee et al. (2011) reported, the oyster shells comprise several distinct layers composed of regular simple prismatic and nacreous or pearl layers. Bulk density is the relevant chemical composition of the limestone. In the present study, the bulk density values of $1.16-1.36 \mathrm{~g} / \mathrm{cm}^{3}$ for limestones were lower than $2.65-2.72 \mathrm{~g} / \mathrm{cm}^{3}$ of limestones in Turkey reported by Kiliç (2013). Palupuh limestones had the lowest bulk density value of $1.16 \mathrm{~g} / \mathrm{cm}^{3}$. On the contrary, Kiliç (2013) said that bulk density increased with an increase in $\mathrm{MgCO}_{3}$ content.

\section{CONCLUSION}

The limestones vary in magnesium level and might be classified as calcite and dolomitic. Oyster shells derived from different habitats determine the magnesium and trace mineral composition. Calcination of limestones increased magnesium concentration and specific density but reduced micro minerals copper and manganese. The calcined oyster shell had a higher zinc concentration, specific density, fine particle portion, but lower bulk and tapped densities, coarse and medium particles than the uncalcined products. The results suggested that the type of limestones and oyster shells determined their thermal decomposition properties.

\section{DECLARATIONS}

\section{Authors' contributions}

Khalil and Andri designed the project, analyzed data, and drafted the manuscript. Khalil and R.K. Rusli prepared and calcinated minerals. R.K. Rusli and Andri edited and corrected the manuscript, Andri organized project implementation and administration. All authors checked the results of the data analysis and agreed to the final draft of the manuscript.

\section{Funding}

This work was a part of the project: Riset Publikasi Bereputasi (RPB) 2021. Andalas University financially supported this study, Grant No. T/12/UN.16.17/PT.01.03/Pangan-RPB/2021.

\section{Acknowledgments}

The authors are grateful to Drs. Sofyan, M.Si, Department. of Animal Nutrition and Feed Technology of IPB University, Bogor, provides laboratory facilities for mineral analysis. This work was a part of the project: Riset Publikasi Bereputasi (RPB) 2021. Andalas University financially supported this study, Grant No. T/12/UN.16.17/PT.01.03/Pangan-RPB/2021. 
The authors would like to thank Dr. Daryoush Babazadeh and Ali Sadeghi for their comments on the draft of this manuscript

\section{Competing interests}

The authors declare no conflict of interest concerning research, authorship, and publication of this article.

\section{Ethical considerations}

Ethical issues (including plagiarism, consent to publish, misconduct, data fabrication and/or falsification, double publication and/or submission, and redundancy) have been checked by the authors.

\section{REFERENCES}

Atomic-Absorption Spectrometry (AAS) (1996). Analytical methods for atomic-absorption Spectroscopy. Perkin-Elmer Corporation, Norwalk, Connecticut, USA. Available at: http://www1.lasalle.edu/ prushan/Intrumental\%20Analysis_files/AAPerkin\%20Elmer\%20guide\%20to\%20all!.pdf

Boynton RS (1980). The chemistry and technology of lime and limestone. 2nd edition. Wiley, New York. Available at: https://www.wiley.com/en-us/Chemistry+and+Technology+of+Lime+and+Limestone\%2C+2nd+Edition-p-9780471027713

Geldart D, Abdullah EC, Hassanpour H, Nwoke LC, and Wouters I (2006). Characterization of powder flowablity using measurement of angle of repose. Chine Particuology, 4: 104-107. Available at: https://www.sciencedirect.com/science/article/abs/pii/S1672251507602474

Gupta P, Arnab D, and Biswas CH (2017). The effect of impurities on the calcination behaviour of CaCO3 nuggets. Journal of Engineering Research, 5(1): 34-45. Available at: https://kuwaitjournals.org/jer/index.php/JER/article/view/1604/1861

Ha S, Lee JW, and Choi SH (2019). Calcination characteristics of oyster shells and their comparison with limestone from the perspective of waste recycling. Journal of Material Cycles and Waste Management, 21(5): 1075-1084. DOI: https://www.doi.org/10.1007/s10163-019-00860-2

Hamester MRR, Balzer PS, and Becker D (2012). Characterization of calcium carbonate obtained from oyster and mussel shells and incorporation in polypropylene. Materials Research, 15(2): 2004-2008. DOI: https://www.doi.org/10.1590/S1516$\underline{14392012005000014}$

Hendronursito Y, Barus J, Amin M, Muttaqii MA, Rajagukguk TO, Isnugroho K, and Birawidha DC (2019). The local mineral potential from East Lampung - Indonesia: The use of basalt rock as a stone meal for cassava plant. Journal of Degraded and Mining Lands Management, 7(1): 1977-1985. DOI: https://www.doi.org/10.15243/jdmlm. 2019.071.1977

Jović M, Mandić M, Šljivić-Ivanović M, and Smičiklas I (2019). Recent trends in application of shell waste from mariculture. Studia Marina, 32(1): 47-62. DOI: https://www.doi.org/10.5281/zenodo.3274471

Kantiranis N, Tsirambides A, Filippidis A, and Christaras B (1999). Technological characteristics of the calcined limestone from Agios Panteleimonas, Macedonia, Greece. Materials and Structures, 32: 546-551. Available at: http://users.auth.gr/ ananias/pdf/Articles\%20in\%20International\%20journals/11\%201999\%20Characteristics\%20calcined\%20li mestone\%20Agios\%20Panteleimonas\%20Mater\%20Struct.pdf

Khalil K, and Anwar S (2007). Studies on the mineral composition of Bukit Kamang' limestone for mineral feed. Media Peteternakan, 30: 18-25. Available at: https://journal.ipb.ac.id/index.php/mediapeternakan/article/view/1020

Khalil K, and Anwar S (2008). Limestone of Bukit Kamang as a calcium source for laying hens. Journal of the Indonesian Tropical Animal Agriculture, 34(3): 174-180. DOI: https://www.doi.org/10.14710/jitaa.34.3.174-180

Khalil K, Primasari B, and Anwar S (2011). Studies on physical properties of Bukit Kamangs' limestone as mineral source for laying hens. Journal of the Indonesian Tropical Animal Agriculture, 36(3): 171-179. DOI: https://www.doi.org/10.14710/jitaa.36.3.171-179

Khalil K, Wati W, Hidayat F, and Evitayani (2018). Physical properties and nutritive values of shell meal derived from different shellfish species and habitats. International Journal of Poultry Science, 17: 116-125. DOI: https://www.doi.org/10.3923/ijps.2018.116.125

Kiliç Ö (2013). Impact of physical properties and chemical composition of limestone on decomposition activation energy. Asian Journal of Chemistry, 25(14): 8116-8120. DOI: http://www.dx.doi.org/10.14233/ajchem.2013.15172

Kurunczi S, Török S, and Chevallier P (2001). A micro-XRF study of the element distribution on the growth front of mussel shell (species of Unio Crassus Retzius). Mikrochimica Acta, 137: 41-48. DOI: http://www.dx.doi.org/10.1007/s006040170026

Lee JW, Choi SH, Kim SH, Cha WS, Kim K, and Moon BK (2018). Mineralogical changes of oyster shells by calcination: A comparative study with limestone. Economic and Environmental Geology, 51(6): 485-492. DOI: http://www.dx.doi.org/10.9719/EEG.2018.51.6.485

Lee SW, Jang YN, and Kim JC (2011). Characteristics of the aragonite layer in adult oyster shells (Crassostrea gigas): Structural study of myostracum including the abductor muscle scar. Evidence-Based Complementary and Alternative Medicine, p. 110. DOI: https://www.doi.org/10.1155/2011/742963

Li M, Yao ZT, Chen T, Lou ZH, and Xia M (2014). The antibacterial activity and mechanism of mussel shell waste derived material. Powder Technology, 264: 577-582. DOI: https://www.doi.org/10.1016/j.powtec.2014.05.067

Moropoulou A, Bakolas A, and Aggelakopoulou E (2001). The effects of limestone characteristics and calcination temperature to the reactivity of the quicklime. Cement and Concrete Research, 31: 633-639. DOI: https://www.doi.org/10.1016/S0008$\underline{8846(00) 00490-7}$ 
Mosher S, Cope WG, Weber FX, Shea D, and Kwak TJ (2012). Effects of lead on Nap, Kp-ATPase and hemolymph ion concentrations in the freshwater mussel Ellptio complanata. Environmental Toxicology, 27(5): 268-276. Available at: https://pubmed.ncbi.nlm.nih.gov/20725939/

Okonkwo PC, and Adefila SS (2013). Investigation of some factors that affect Jakura limestone burning. African. Journal of Pure and Applied Chemistry, 7(8): 280-290. DOI: https://www.doi.org/10.5897/AJPAC2013.0513

Özer AK (2003). The characteristics of phosphate rock for upgrading in a fluidized bed. . Advanced Powder Technology, 14(1): 3342. DOI: https://www.doi.org/10.1163/156855203762469885

Park K, Sadeghi K, Thanakkasaranee S, Park YI, Park J, Nam KH, Han H, and Seo J (2021). Effects of calcination temperature on morphological and crystallographic properties of oyster shell as biocidal agent. Applied Ceramic Technology, 18(2): 302-311. DOI: https://www.doi.org/10.1111/ijac.13647

Ruttloff C (1981). Technologie Mischfuttermittle. VEB Fachbuchverlag, Leipzig, pp. 76-77.

Sari N, Jalil Z, and Rahwanto A (2013). Identification of oxide compound in dolomite mineral from Aceh Tamiang Region. Journal of Aceh Physics Society, 2(1): 1-2. Available at: http://www.jurnal.unsyiah.ac.id/JAcPS/article/view/687

Silveira MACW, da Luz JAM, de Faria GL, and Coutinho FMP (2020). Calcination thermokinetics of three Brazilian limestones. Cerâmica, 66: 297-306. DOI: http://www.dx.doi.org/10.1590/0366-69132020663792910

Steel RGD, Torrie JH, and Dicky JH (1997). Principles and procedures of statistics: A biometritrical approach. ${ }^{\text {rd }}$ Edition. McGrawHill Book, New York, USA, pp. 173-343.

Yao Z, Xia M, Li H, Chen T, Ye Y, and Zheng H (2014). Bivalve shell: not an abundant useless waste but a functional and versatile biomaterial. Critical Reviews in Environmental Science and Technology, 44(22): 2502-2530. DOI: https://www.doi.org/10.1080/10643389.2013.829763 